\title{
Rapid Quantification of Trimethylamine
}

\author{
Zheng Li, Hao Li, Maria K. LaGasse and Kenneth S. Suslick*,
}

Department of Chemistry, University of Illinois at Urbana-Champaign, 600 S. Mathews Avenue, Urbana, Illinois 61801, USA

E-mail: ksuslick@illinois.edu

\section{Table of Contents}

1. Materials and Methods section

2. Experimental section

3. Sensor response graphs

4. Statistical analysis

5. Raw data 


\section{Materials and Methods}

Array preparation. All reagents were analytical-reagent grade, purchased from Sigma-Aldrich and used as received. Preparation and printing of colorimetric sensor arrays have been described in detail elsewhere. ${ }^{1}$ The chemoresponsive dyes used in each spot is elaborated in Table S1 along with a color-coded legend indicating the expected chemical reactivity of each spot; visualized image captured by handheld device is shown in Fig. S1.

Analytes generation. All gases at their selected concentrations were prepared by mixing the gas stream of prediluted analyte with dry and wet nitrogen gas using MKS digital mass-flow controllers to achieve the desired concentrations and relative humidity at a total flow rate of $500 \mathrm{sccm}$ (Figure S2). Gas flow was running for 30 min to achieve a stabilized concentration before each measurement. Analytes concentrations were confirmed by in-line analysis with FTIR using a MKS multigas analyzer (model 2030). Solutions of TMA and other amines were prepared in $1 \mathrm{X}$ PBS $(10 \mathrm{mM}, \mathrm{pH} 7.4)$ by spiking the corresponding amount of solutes into the stock buffer solution to achieve the desired vapor or liquid concentrations.

Data obtained on flatbed scanner. The arrays were imaged as a function of time on an ordinary flatbed scanner (Epson Perfection V600); the before-exposure image was acquired after 2 min exposure of wet $\mathrm{N}_{2}$ at $50 \%$ relative humidity or blank buffer; after-exposure images were acquired with full equilibration after exposure to the targeted vapor or aqueous concentrations. Difference maps were obtained by subtracting the red, green, and blue (RGB) values of before-exposure images from those of after-exposure images; the diameter of each sensor spot was $\sim 50$ pixels, the values of which were averaged. Color differences was digitized using a customized software package, SpotFinder 1.0.6 (iSense LLC., Mountain View, CA). Septuplicate trials were taken for each analyte or concentration.

Data obtained on iPhone 5s. Sensor array images were collected using the camera of an Apple iPhone 5s. The same exposure procedures and data process protocols used for the flatbed scanner were applied to the cell phone detection, except that RGB values of each sensor element were corrected using the equation $R_{\text {corr }}=R_{i} / R_{\text {white }} *$ 255 , in which $R_{i}$ is the initial RGB value of the sensor element while $R_{\text {white }}$ is the RGB value of the white reference; white reference for each sensor element is taken from the white blank area on each side of the sensor element. The correction method is used to minimize inconsistencies of brightness from image to image due to changes in ambient lighting, as well as the inconsistency of lighting among sensor elements within each single image caused by non-uniform illumination across the array.

Data obtained on handheld reader. The experimental setup using the handheld imaging device is shown in Figure S2. The construction and specifications of the handheld device used in this study have been elaborated in recent publications. ${ }^{2}$ Raw data was normalized using a calibration created from a one-time measurement of a $0 \%$ reflectance standard (i.e., the sensor array with all LEDs turned off) and a 100\% reflectance standard (i.e., a white blank array). For visualization, difference maps were constructed by taking the absolute value of the reflectance measurements before and during 2 min exposure and scaling a relevant color range to the 8-bit color scale (i.e., 0-255); for all statistical analyses, the actual values of the reflectance measurements were used without modification. For S/N measurements, signal and noise were calculated for each data channel using all trials in the data set (i.e., red, green, and blue values of 20 sensor elements; 60 dimensions in total); signals for each channel were defined as the difference between each analyte trial measurement and the average of the nonexposed controls, and noise was defined as the standard deviation among the control data; septuplicate trials were taken for each analyte or concentration.

Database analysis. The chemometric analysis was performed on the color difference vectors using the MultiVariate Statistical Package ${ }^{\mathrm{TM}}$ (MVSP v.3.1, Kovach Computing); in all cases, minimum variance (i.e., "Ward's Method") was used for HCA clustering. Support vector machine (SVM) analysis was carried out using a leaveone-out permutation method based on an open-source SVM library, LIBSVM. ${ }^{3}$ 
Table S1. Sensor spot compositions of the colorimetric sensor array.

\begin{tabular}{|c|l|c|}
\hline Spot \# & \multicolumn{1}{|c|}{ Name } & $\mathrm{mg}$ \\
\hline 1 & Ethanone + TsOH & $2 / 10 \mu \mathrm{L}$ \\
\hline 2 & $\alpha$-Naphthyl Red + TsOH & $4 / 20 \mu \mathrm{L}$ \\
\hline 3 & $5,10,15,20$-tetrakis(2,4,6-trimethylphenyl)porphyrinatozinc(II) & 4.0 \\
\hline 4 & Tetraiodophenolsulfonephthalein & 4.0 \\
\hline 5 & Fluorescein & 2.0 \\
\hline 6 & Bromocresol Green & 4.0 \\
\hline 7 & Methyl Red & 4.0 \\
\hline 8 & Bromocresol Purple & 4.0 \\
\hline 9 & Bromophenol Red & 4.0 \\
\hline 10 & Rosolic Acid & 4.0 \\
\hline 11 & Bromopyrogallol Red & 2.0 \\
\hline 12 & Pyrocatechol Violet & 4.0 \\
\hline 13 & $4-[2-[4-($ dimethylamino)phenyl]ethenyl]-2,6-dimethylpyrylium & 2.0 \\
\hline 14 & LiNO $_{3}+$ Cresol Red & $15 / 4$ \\
\hline 15 & ${\mathrm{~Pb}(\mathrm{OAc})_{2}+\text { Disperse Red }}_{2}$ & $15 / 0.5$ \\
\hline 16 & $\mathrm{AgNO}_{3}+$ Bromophenol Blue & $5.0 / 2.0$ \\
\hline 17 & $\mathrm{AgNO}_{3}+$ Bromocresol Green & $5.0 / 2.0$ \\
\hline 18 & ${\mathrm{Zn}\left(\mathrm{OAc}_{2}+m \text {-Cresol Purple }+ \text { TBAH }\right.}_{2}$ & $20 / 4 / 50 \mu \mathrm{L}$ \\
\hline 19 & $\mathrm{HgCl}_{2}+$ Bromophenol Blue + TBAH & $5.0 / 4.0 / 50 \mu \mathrm{L}$ \\
\hline 20 & $\mathrm{HgCl}_{2}+$ Bromocresol Green + TBAH & $5.0 / 4.0 / 50 \mu \mathrm{L}$ \\
\hline & $\mathrm{TBAH}_{1} 1.0 \mathrm{M}$ in 2-MeOEtOH & \\
\hline & $\mathrm{TsOH}_{1}$ 1.0 M in 2-MeOEtOH & \\
\hline & & \\
\hline
\end{tabular}
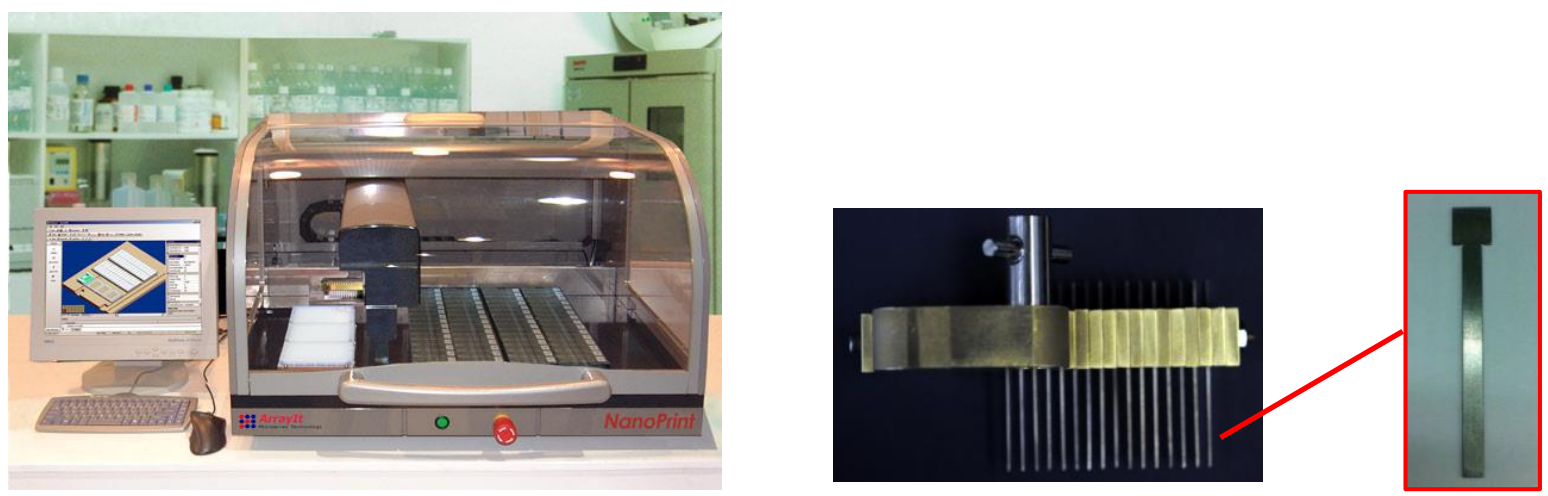

Figure S1. Array-it Nano Printer used to print array cartridges (left) and rectangular pin-holder and pins for printing (right). 


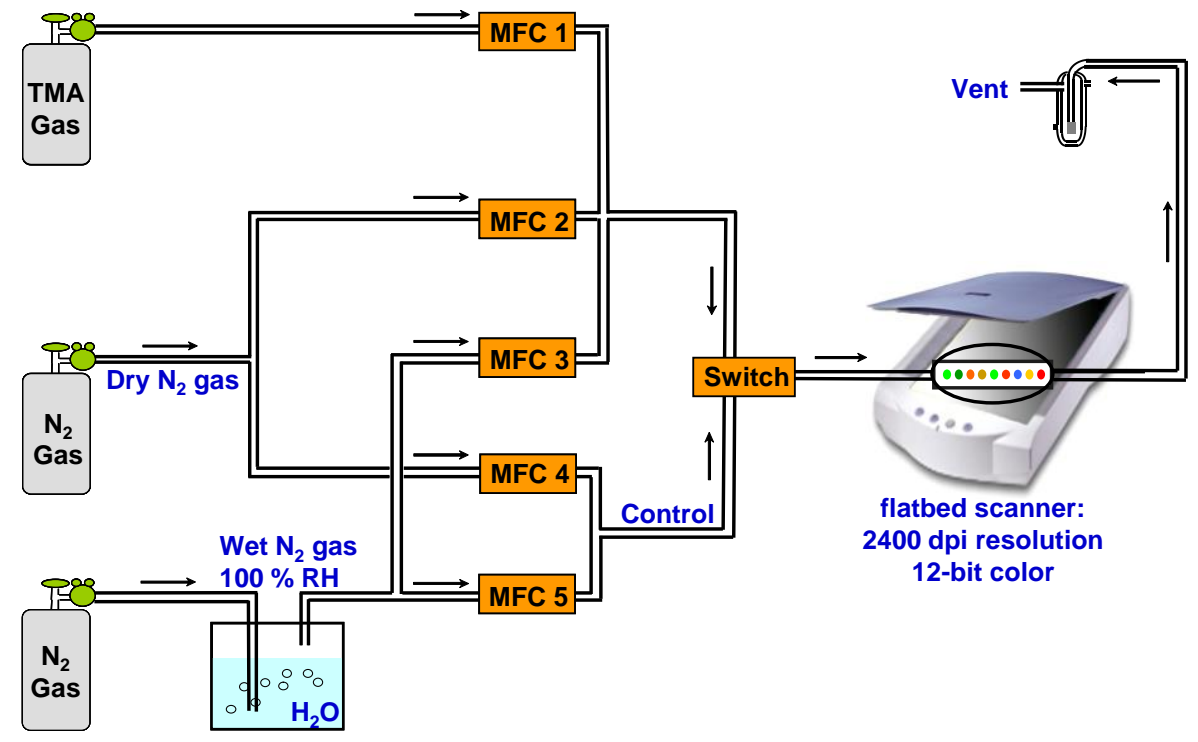

Figure S2. Gas mixing apparatuses for exposure of sensor array to gaseous TMA. MFC = mass flow controller.

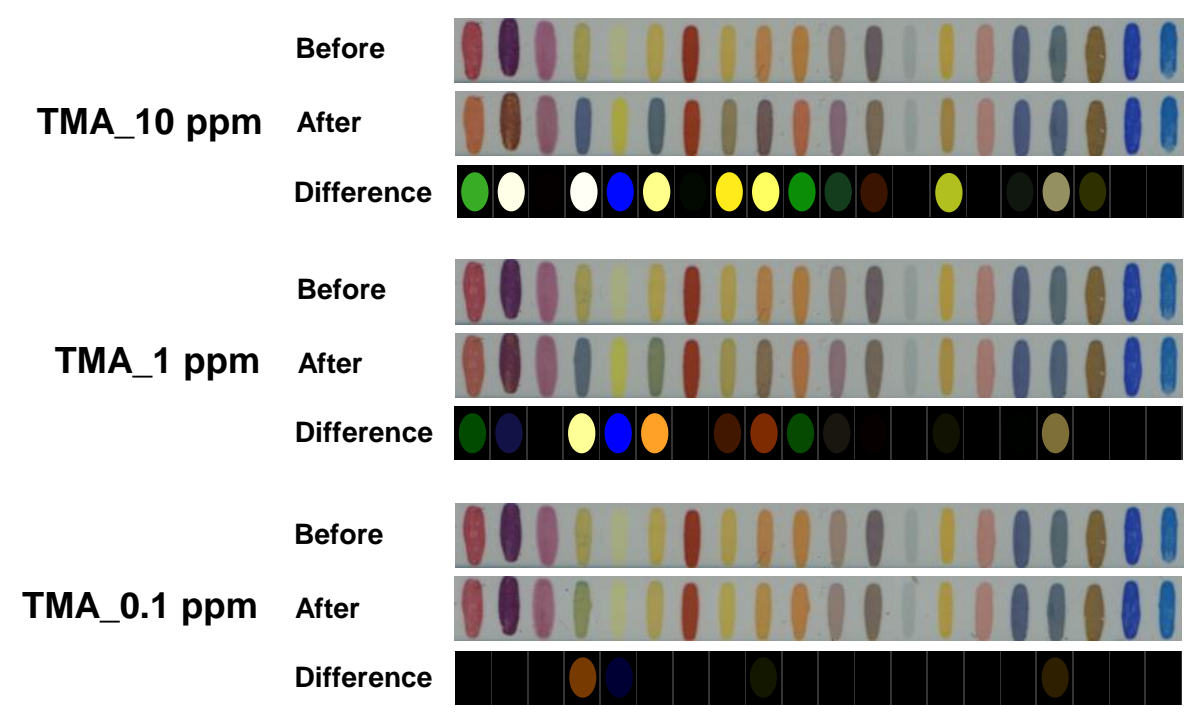

Figure S3. Representative images before and after exposure and color difference images for 2 min exposure to 10,1 and 0.1 ppm TMA. For visualization, the color difference images are shown for a color range expanded from 4 bits (4-19) to 8 bits (0-255). 

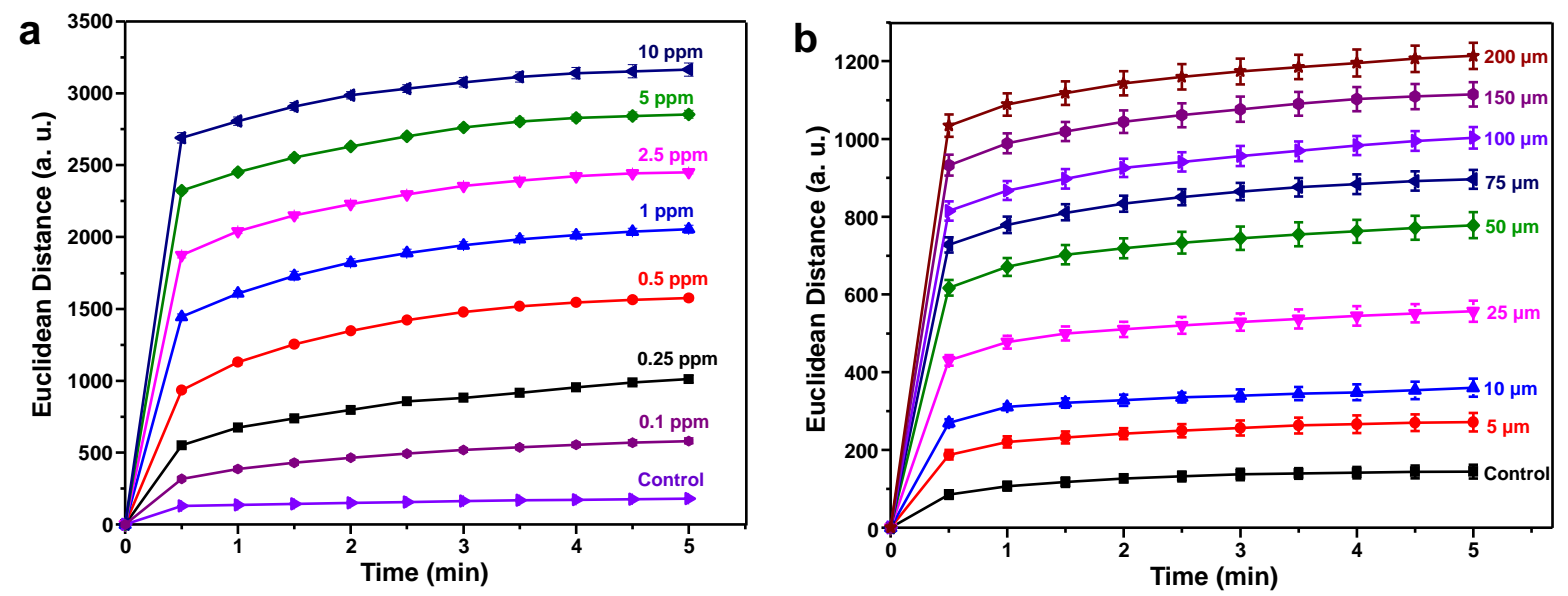

Figure S4. Array response curves of (a) gaseous and (b) aqueous TMA concentrations as a function of time. The average value with error bars set to $2 \sigma$ from septuplicate trials is shown. Total Euclidean distance is the total length of the color difference vector, i.e., the total array response, defined as the square root of the sum of the squares of the changes in RGB values of all 60 dimensions. The average value with error bars set to $2 \sigma$ from septuplicate trials for each concentration is shown.
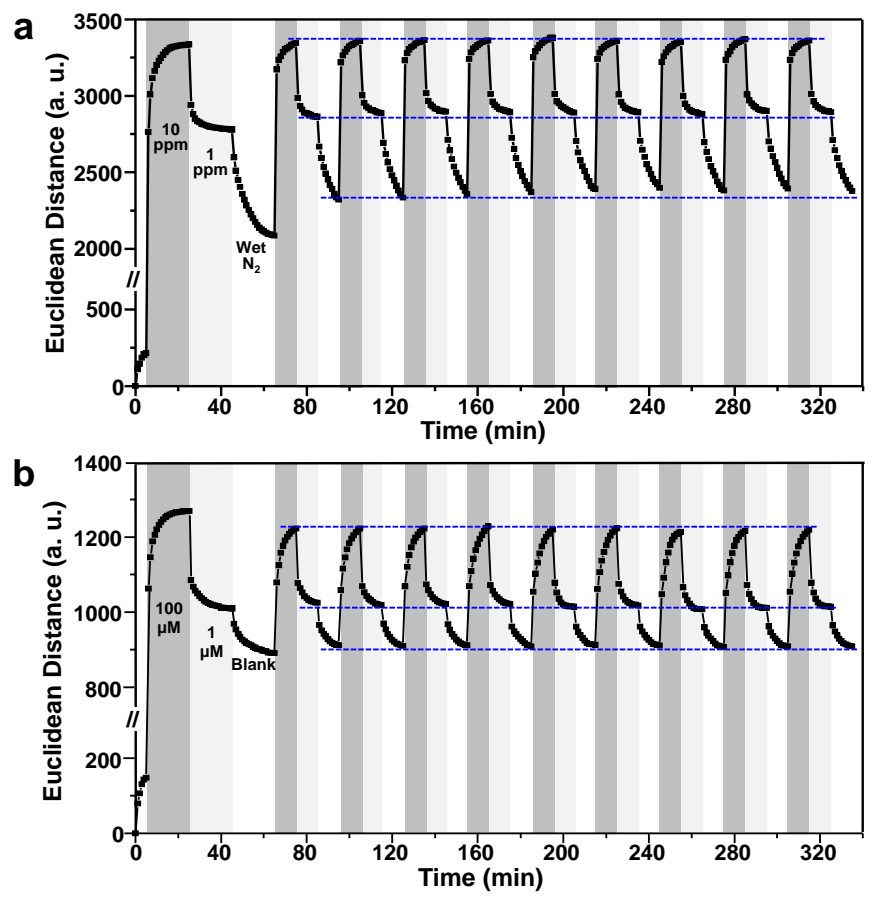

Figure S5. Reversibility of sensor array response during 10 rounds of cycling. (a) Gaseous TMA exposure of a single array from nitrogen to $10 \mathrm{ppm}$, and then repeatedly from $10 \mathrm{ppm}$ to $1 \mathrm{ppm}$ and back to the background (i.e., nitrogen at $50 \%$ relatively humidity). (b) Aqueous TMA exposure of a single array from the blank buffer to $100 \mu \mathrm{M}$, and then repeatedly from $100 \mu \mathrm{M}$ to $1 \mu \mathrm{M}$ and back to the blank. Data were acquired every $1 \mathrm{~min}$; total response duration is $335 \mathrm{~min}$; the total array response is represented by the total Euclidean distance of the 60 -dimensional color difference vector. $90 \%$ of complete equilibration is achieved within $2 \mathrm{~min}$ of the first exposure to $10 \mathrm{ppm}$ TMA and within $3 \mathrm{~min}$ for subsequent cycling between 10 and $1 \mathrm{ppm}$ exposures. The blue lines are horizontal showing the lack of drift over 10 rounds of cycling. 

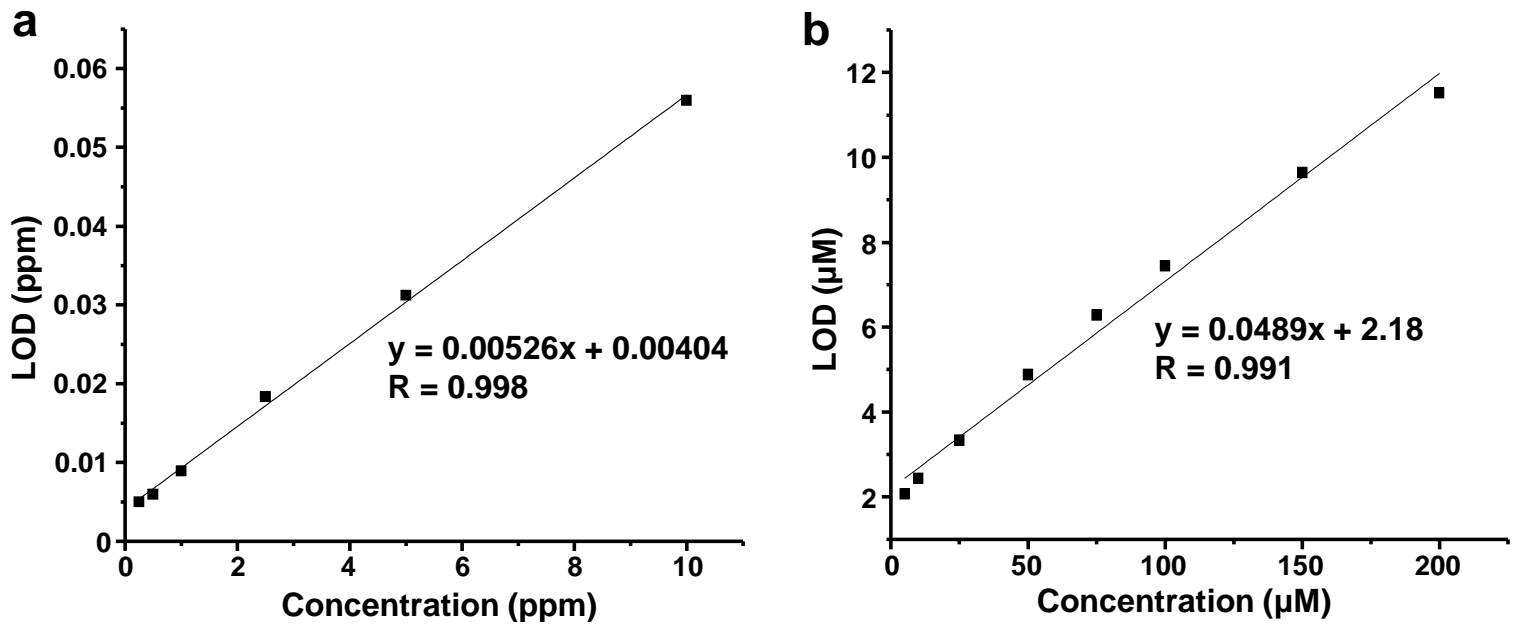

Figure S6. Calibration curves for extrapolation of the LOD of (a) gaseous and (b) aqueous TMA. Concentration ranges from $0.1 \mathrm{ppm}$ to $10 \mathrm{ppm}$ for gases and $5 \mu \mathrm{M}$ to $200 \mu \mathrm{M}$ for solutions. LOD of TMA is calculated to be 4 ppb in the gas and $2 \mu \mathrm{M}$ in the solution.
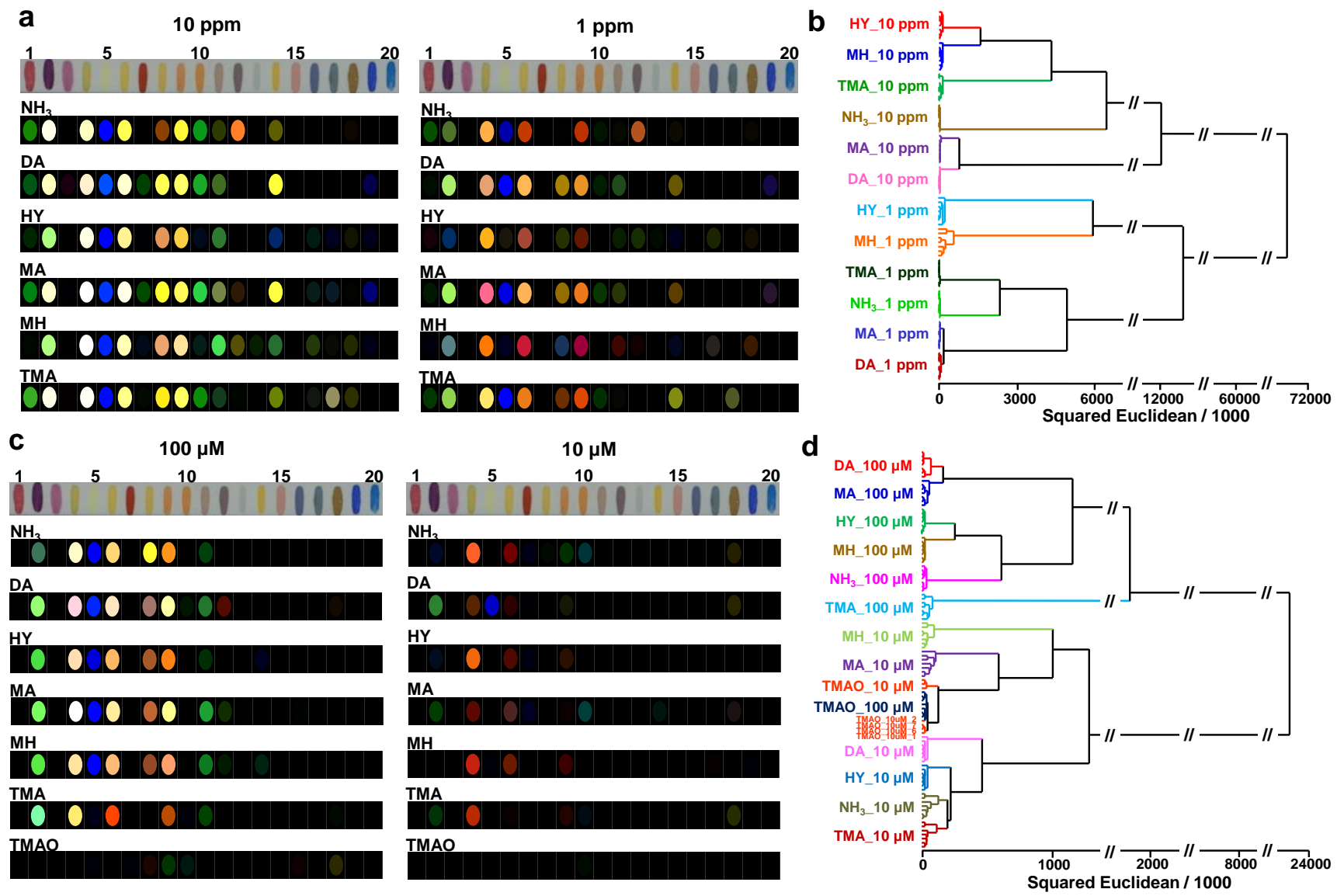

Figure S7. (a) Average response of the sensor array to gaseous amines at $10 \mathrm{ppm}$ and 1 ppm. (b) Dendrogram of hierarchical cluster analysis for gaseous amines at two concentrations. (c) Average response of the sensor array to aqueous amines at $100 \mu \mathrm{M}$ and $10 \mu \mathrm{M}$. (d) Dendrogram of hierarchical cluster analysis for aqueous amines at two concentrations. Each sample was repeated in septuplicate trials. For visualization of the difference images, the color range is expanded from 4 to 8 bits per color (i.e., RGB color range of 4-19 expanded to 0-255). 

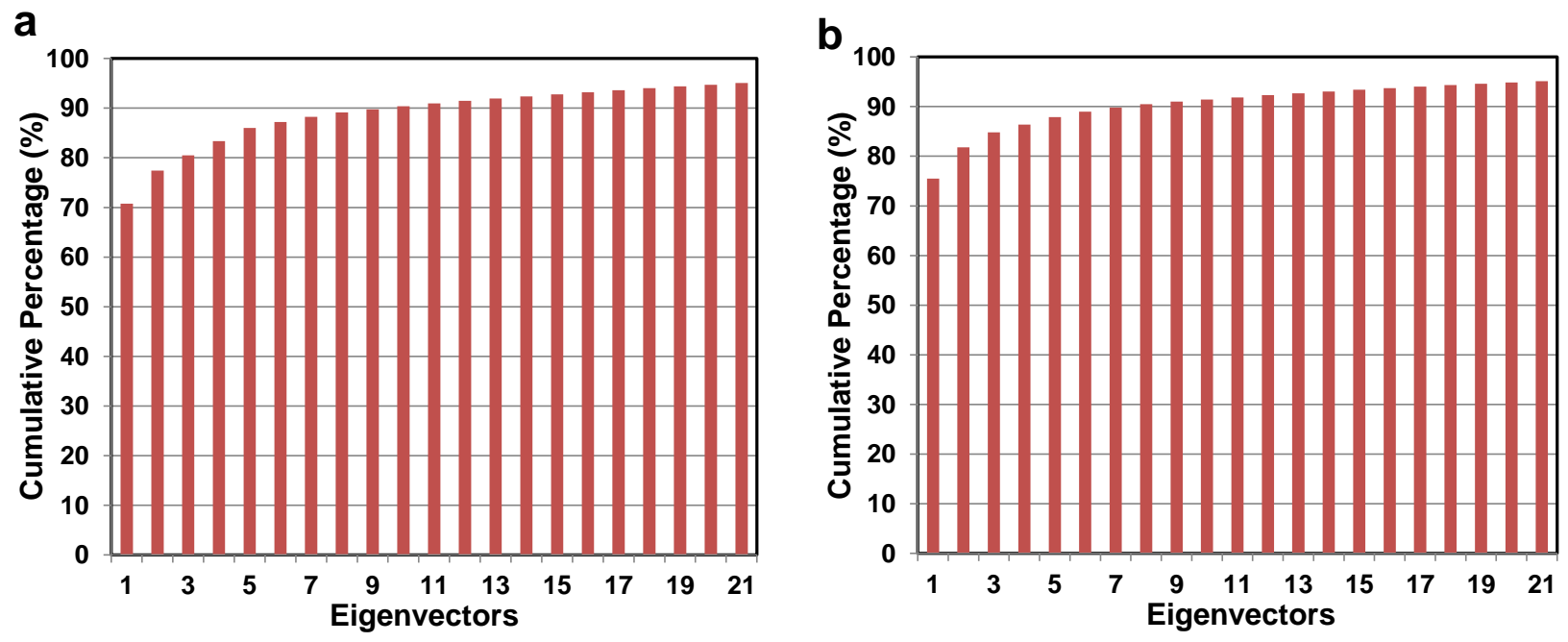

Figure S8. Scree plot from a principal component analysis of (a) all gaseous amines at $10 \mathrm{ppm}$ and $1 \mathrm{ppm}$ and (b) all aqueous amines at $100 \mu \mathrm{M}$ and $10 \mu \mathrm{M}$. 21 dimensions are required to capture $95 \%$ of the total variance in both gaseous and aqueous cases, consistent with the wide range of analyte chemical properties probed by the sensor array.
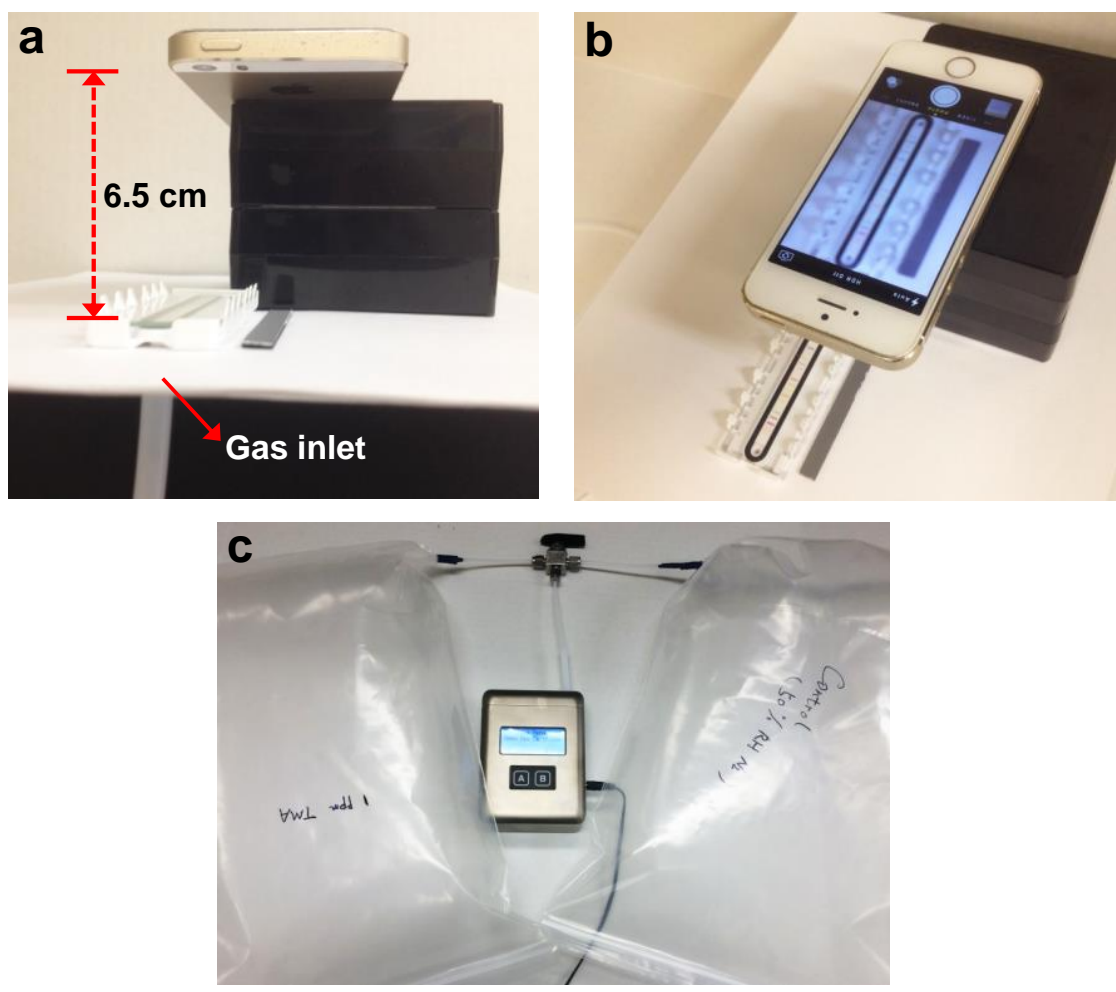

Figure S9. Imaging set-ups using a cellphone or a handheld analyzer. (a) Side view of the cellphone scanning platform. iPhone is $6.5 \mathrm{~cm}$ above the colorimetric sensor array. (b) Top view of the cellphone platform. (c) Handheld analyzer detection. The handheld device samples gas from polyethylene bags either (right) containing 50\% RH nitrogen or (left) TMA at a premixed concentration in 50\% RH nitrogen. 

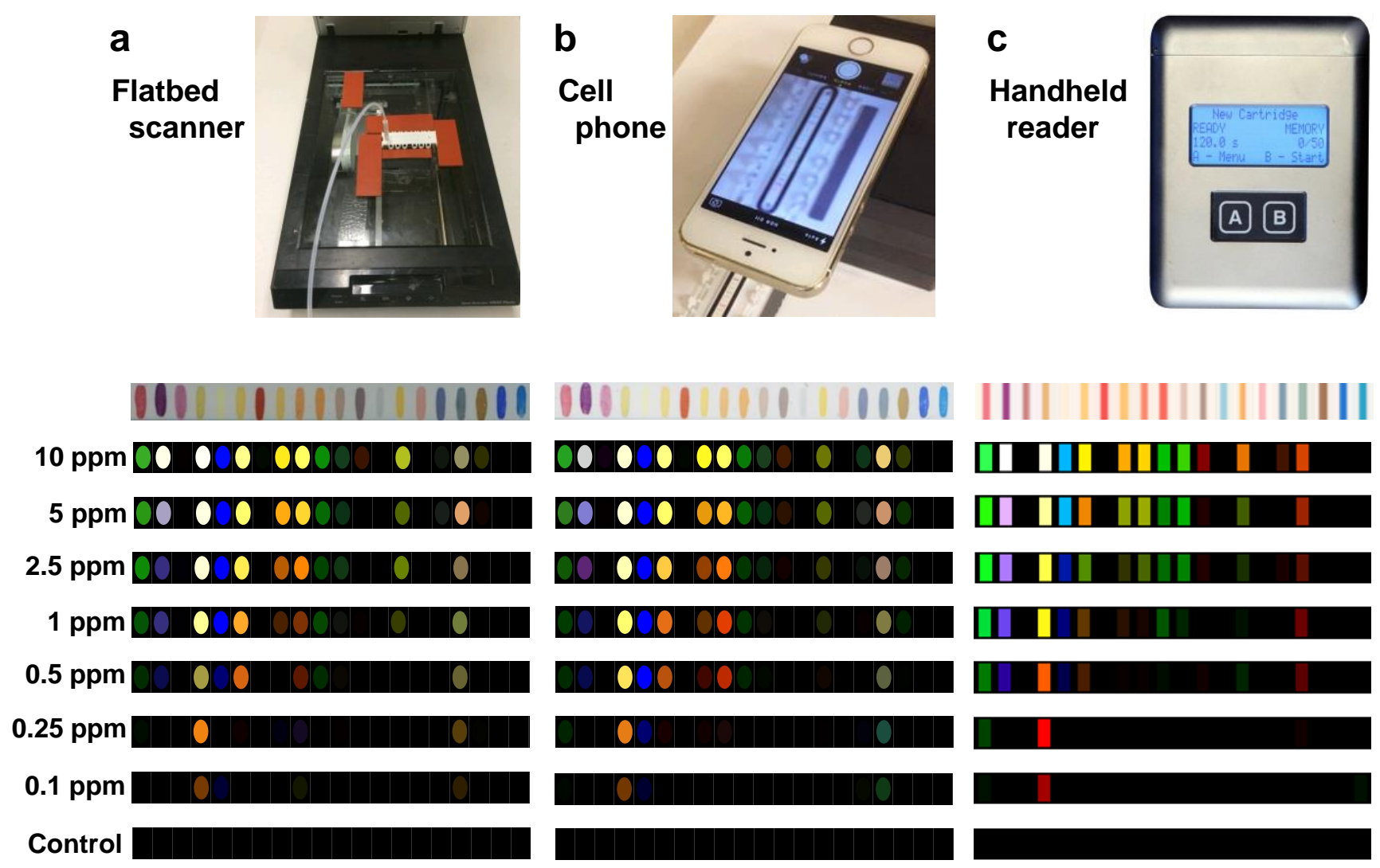

Figure S10. Average sensor array responses of septuplicate trials after 2 min exposure to various concentrations of TMA collected by (a) flatbed scanner, (b) iPhone 5s camera, or (c) handheld reader. For visualization purposes, the color range is expanded from 4 to 8 bits per color (i.e., RGB color range of 4-19 expanded to 0-255). 

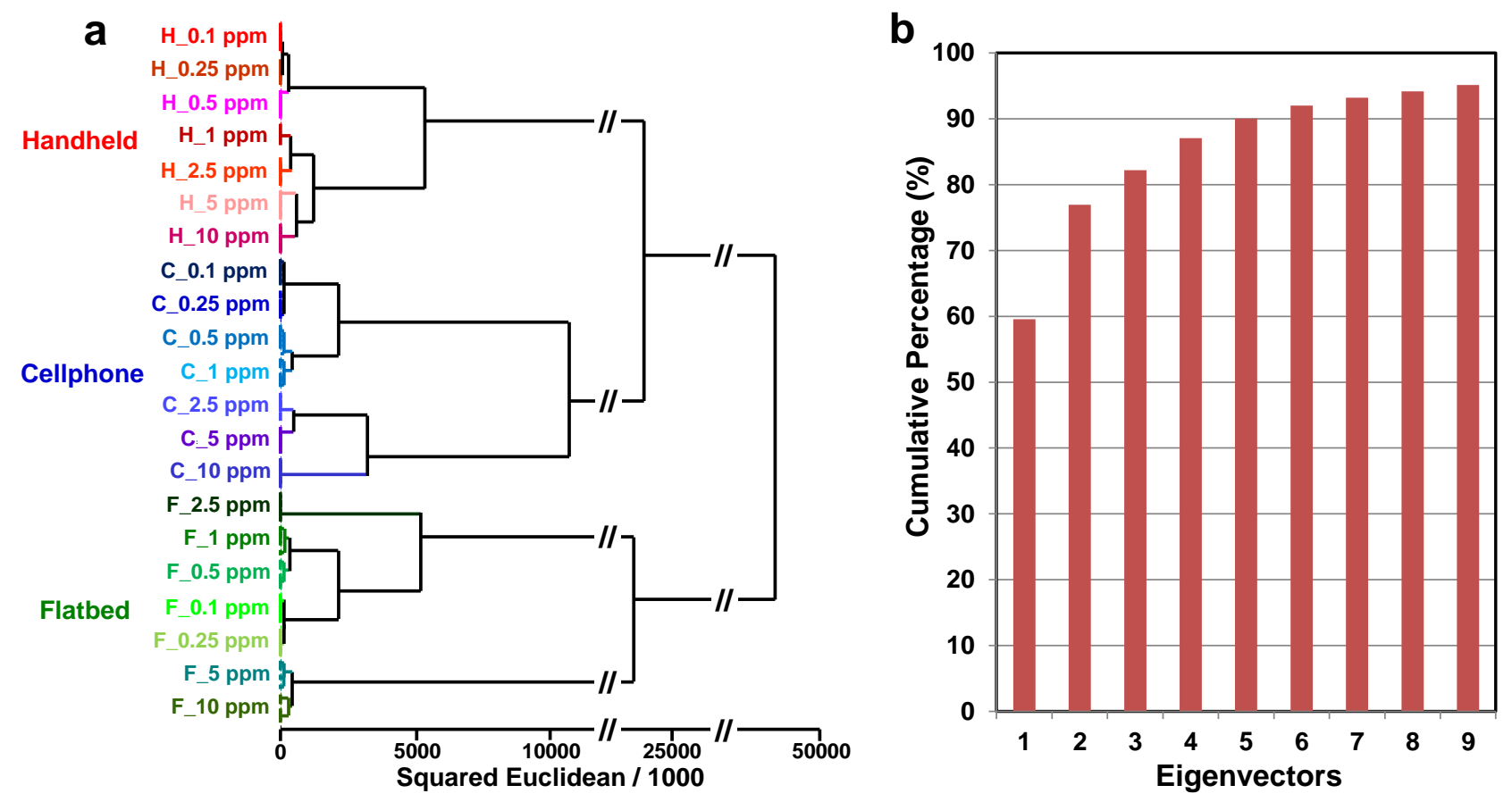

Figure S11. (a) Dendrogram of hierarchical cluster analysis and (b) scree plot of principal component analysis on the database of seven concentrations of TMA vapors collected by three scanning devices.
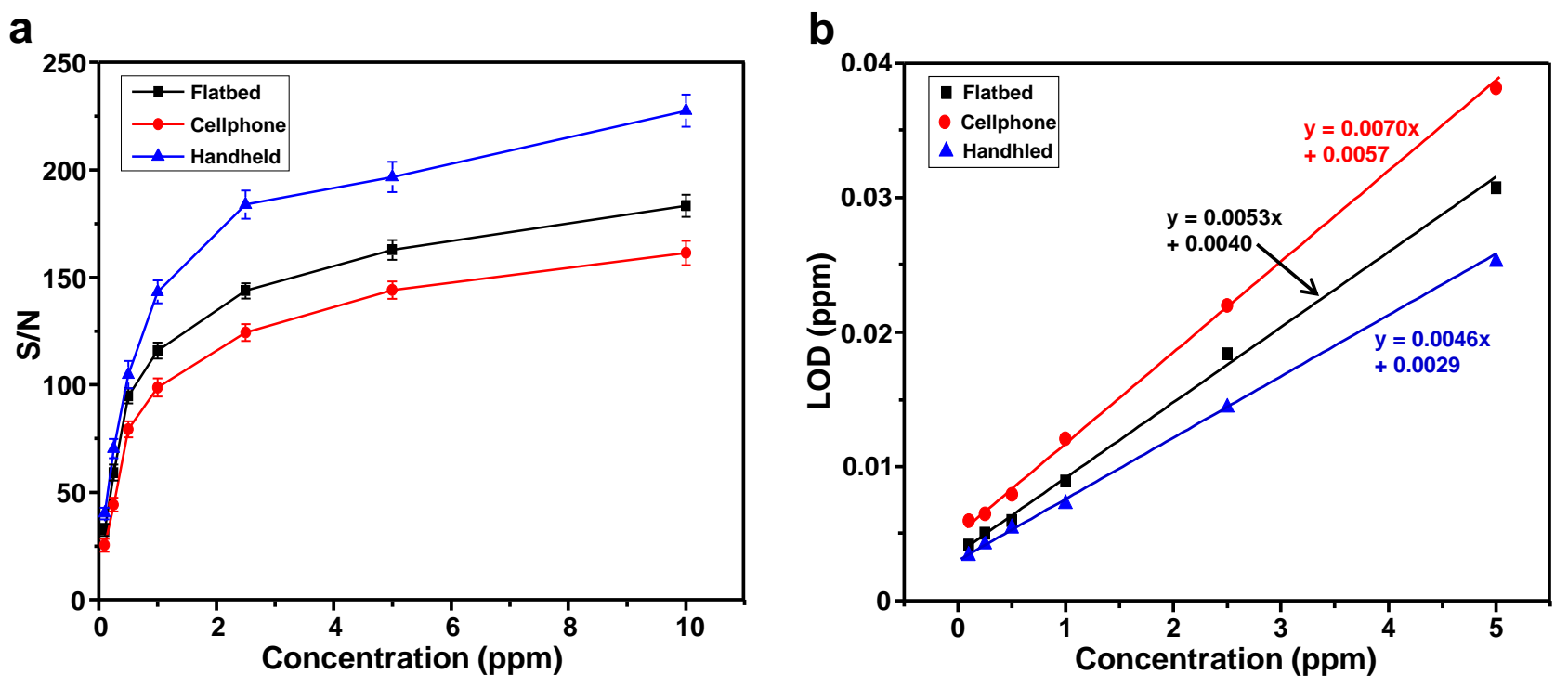

Figure S12. (a) Signal to noise ratios from the most responsive RGB channels observed in flatbed or iPhone detection as a function of TMA concentrations. The average value with error bars set to $2 \sigma$ from quintuplicate trials is shown. (b) Calibration curves for extrapolation of the LOD of TMA vapors obtained by the three imaging devices. LOD of TMA vapors is calculated to be $3 \mathrm{ppb}$ for the handheld analyzer, $4 \mathrm{ppb}$ for the flatbed scanner and $6 \mathrm{ppb}$ for the iPhone. 

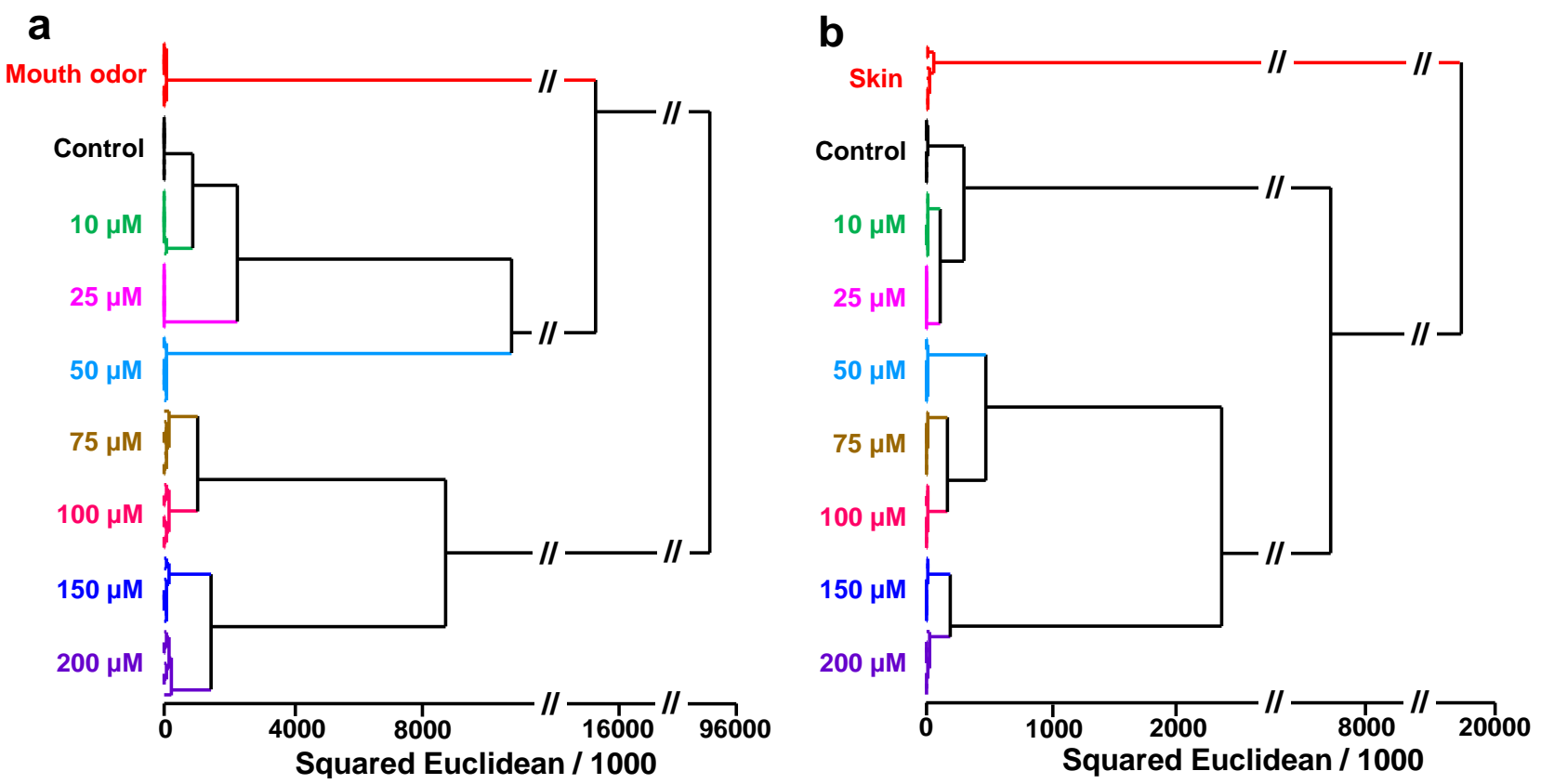

Figure S13. Dendrogram of hierarchical cluster analysis for (a) dataset of simulated mouth odor test and (b) dataset of simulated skin odor test.

\section{References}

(1) Askim, J. R.; Li, Z.; LaGasse, M. K.; Rankin, J. M.; Suslick, K. S., Chem. Sci. 2016, 7, 199-206.

(2) Askim, J. R.; Suslick, K. S., Anal. Chem. 2015, 87, 7810-7816.

(3) Chang, C.-C.; Lin, C.-J., ACM Trans. Intell. Syst. Technol. 2011, 2, 1-27. 
Table S2. SVM Cross-validation Results.
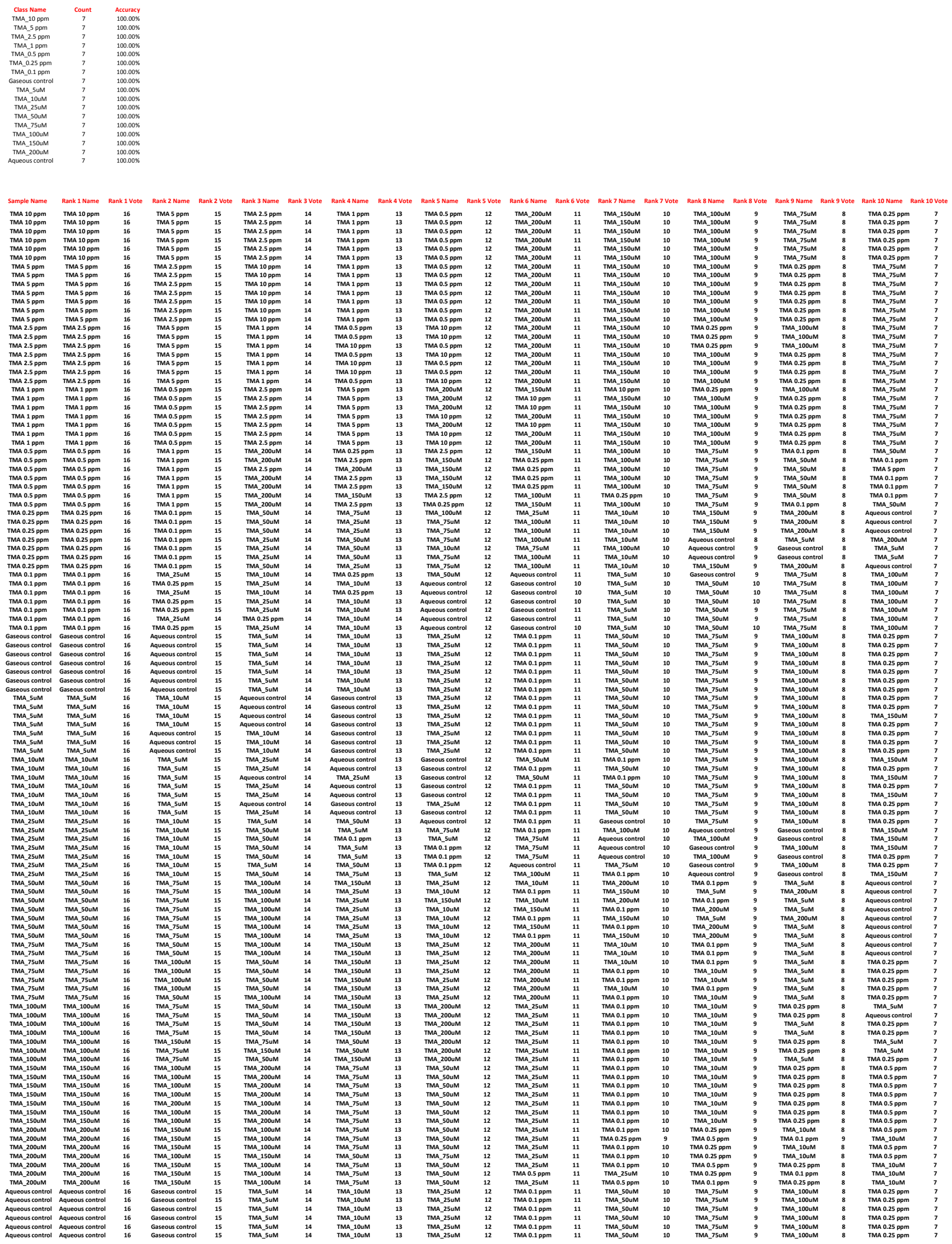


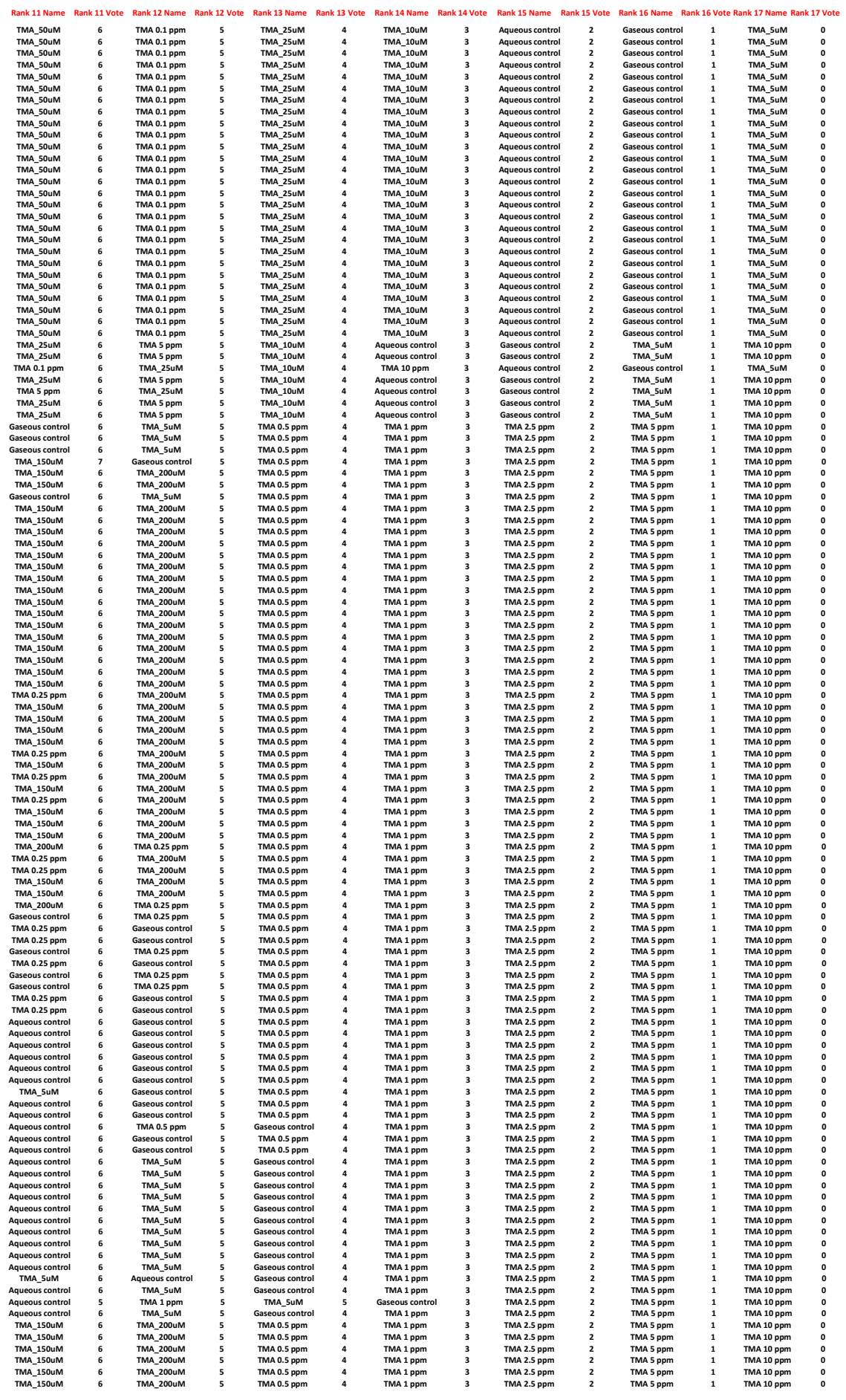


Table S3. Database for different concentrations of gaseous or aqueous TMA after two min exposure using flatbed scanner.

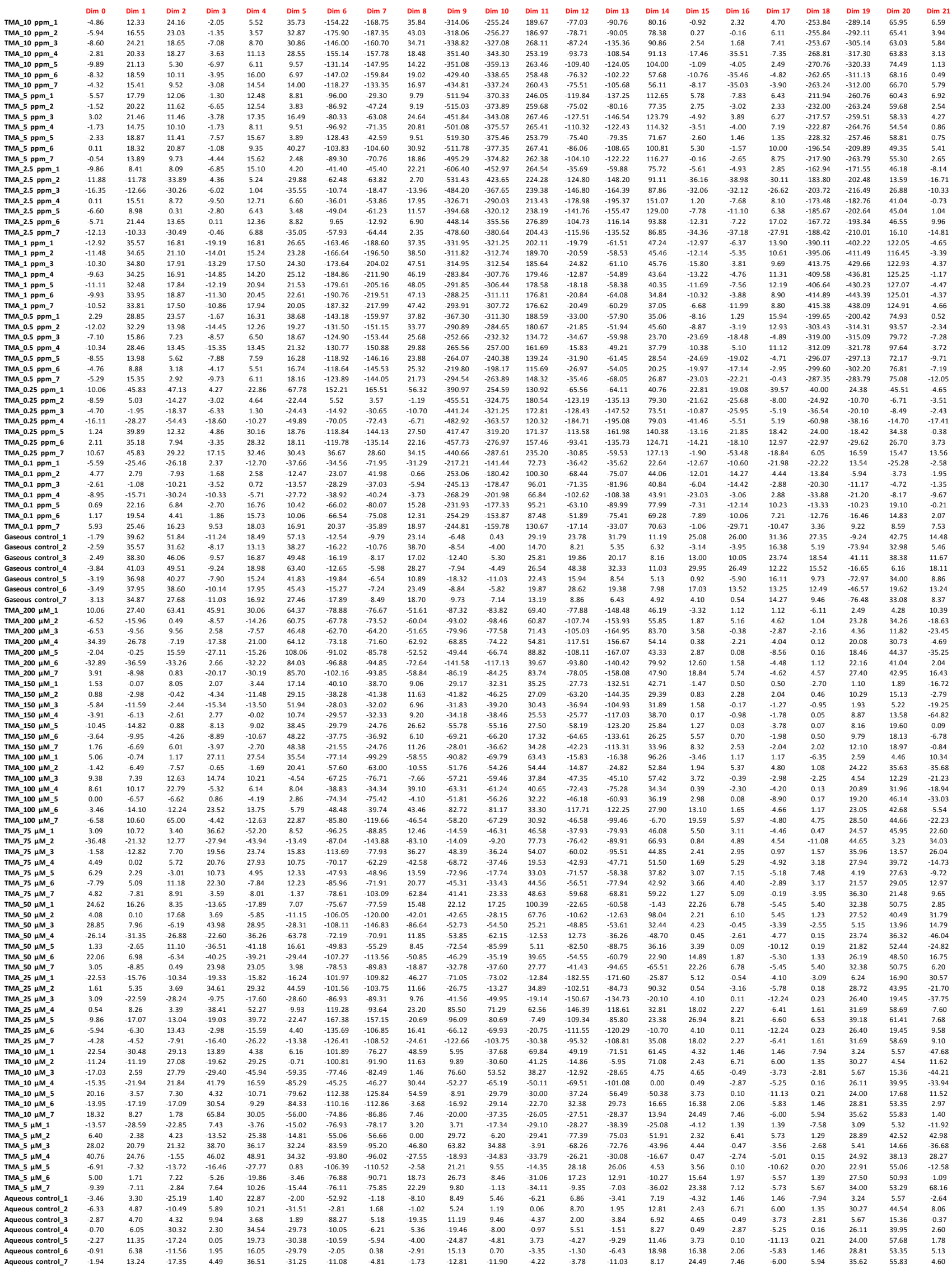




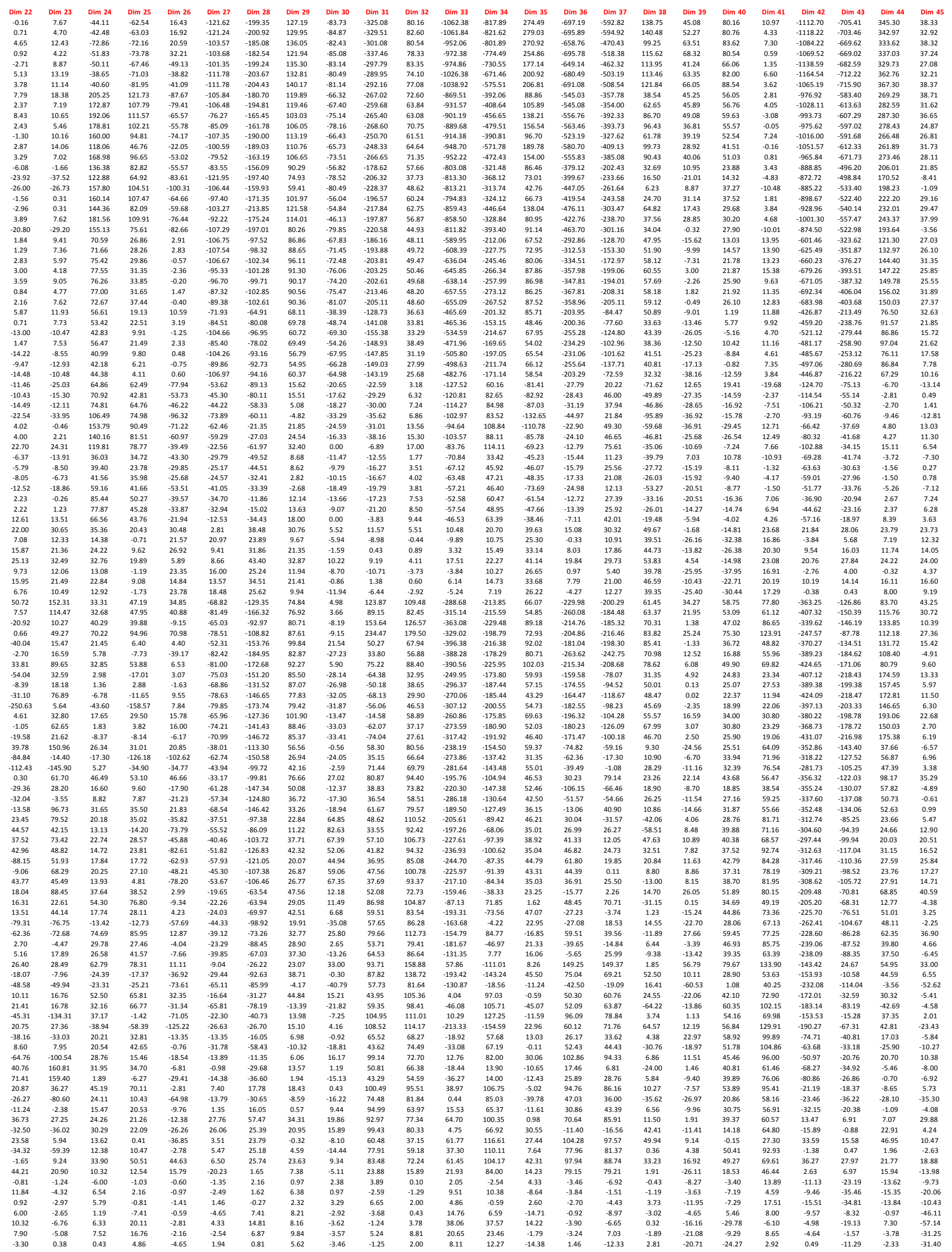




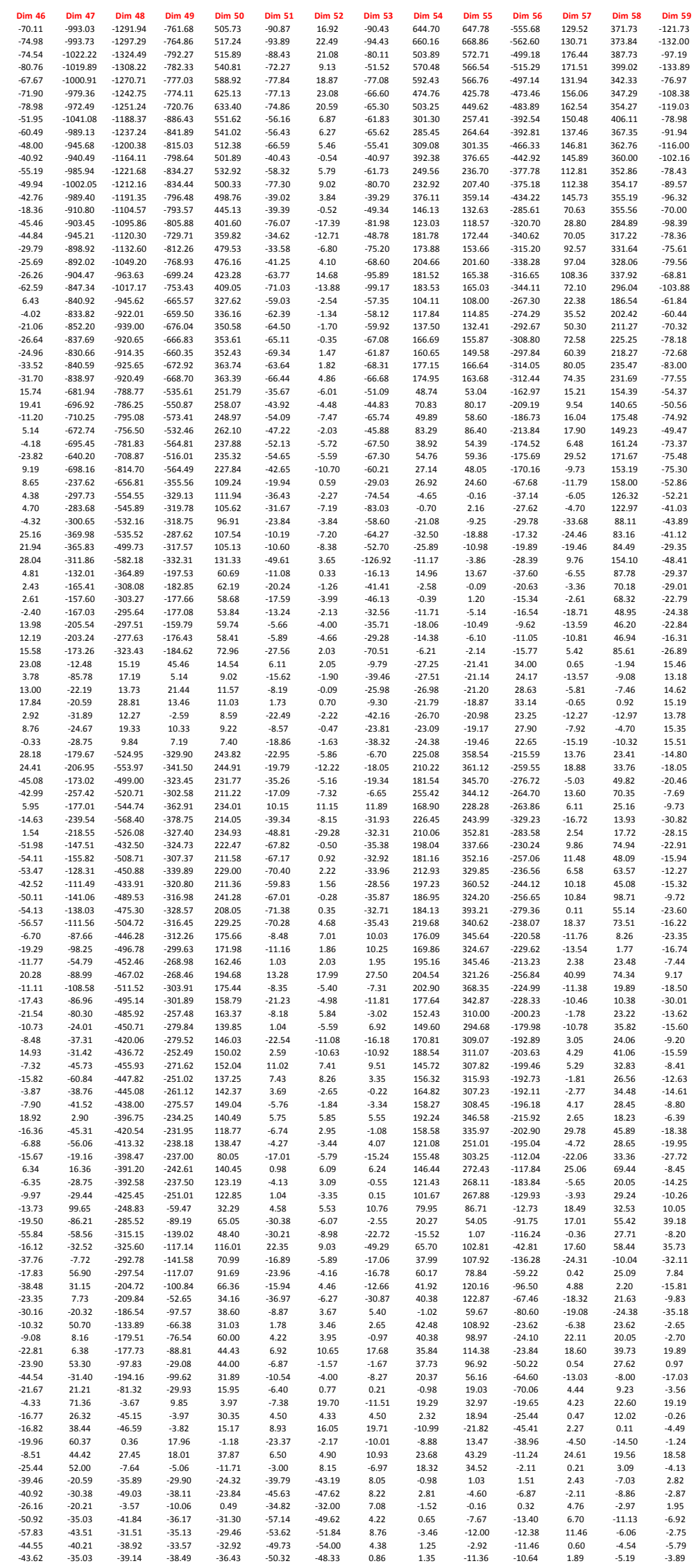


Table S4. Database for two min exposure to other amines at two concentrations using flatbed scanner.

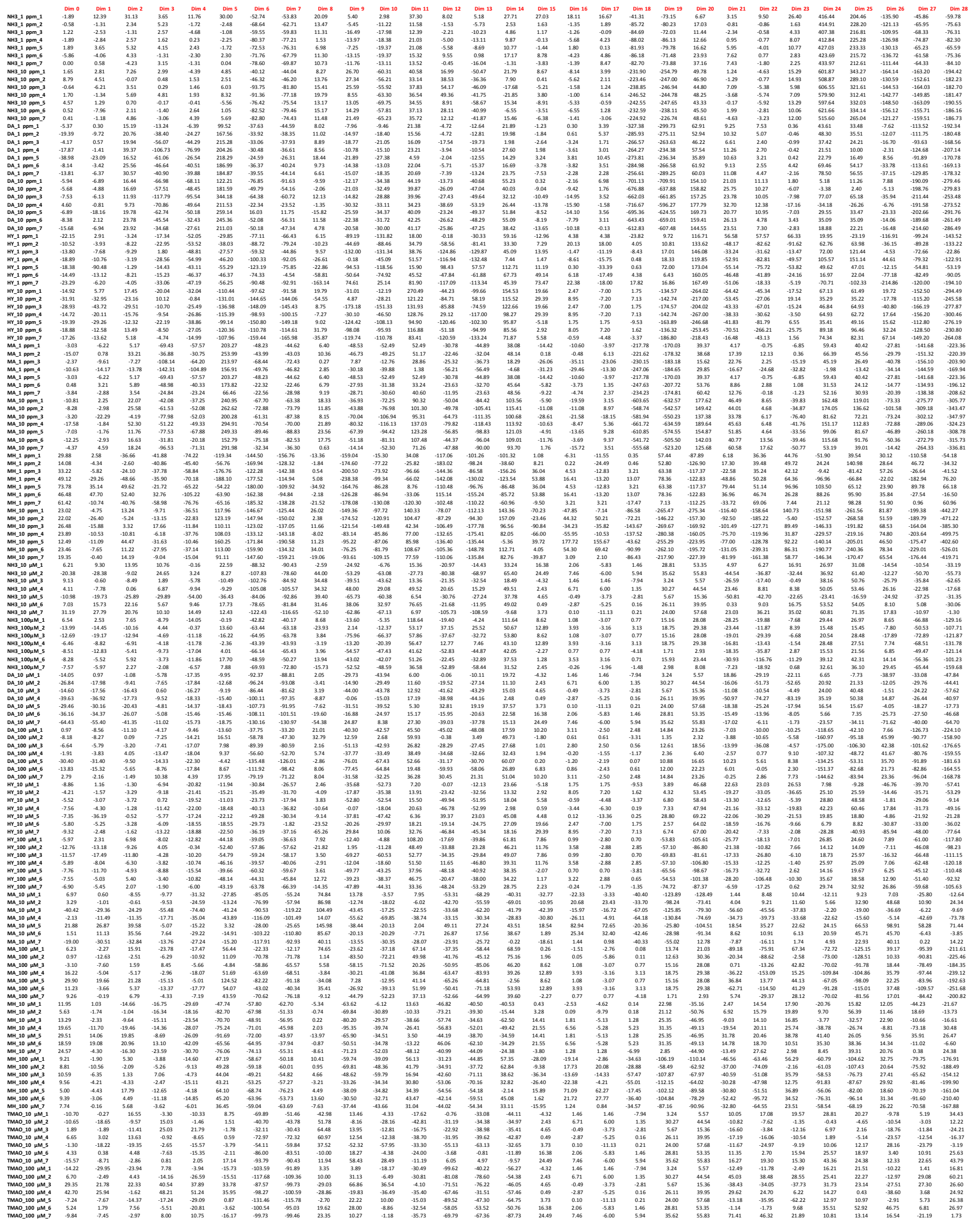




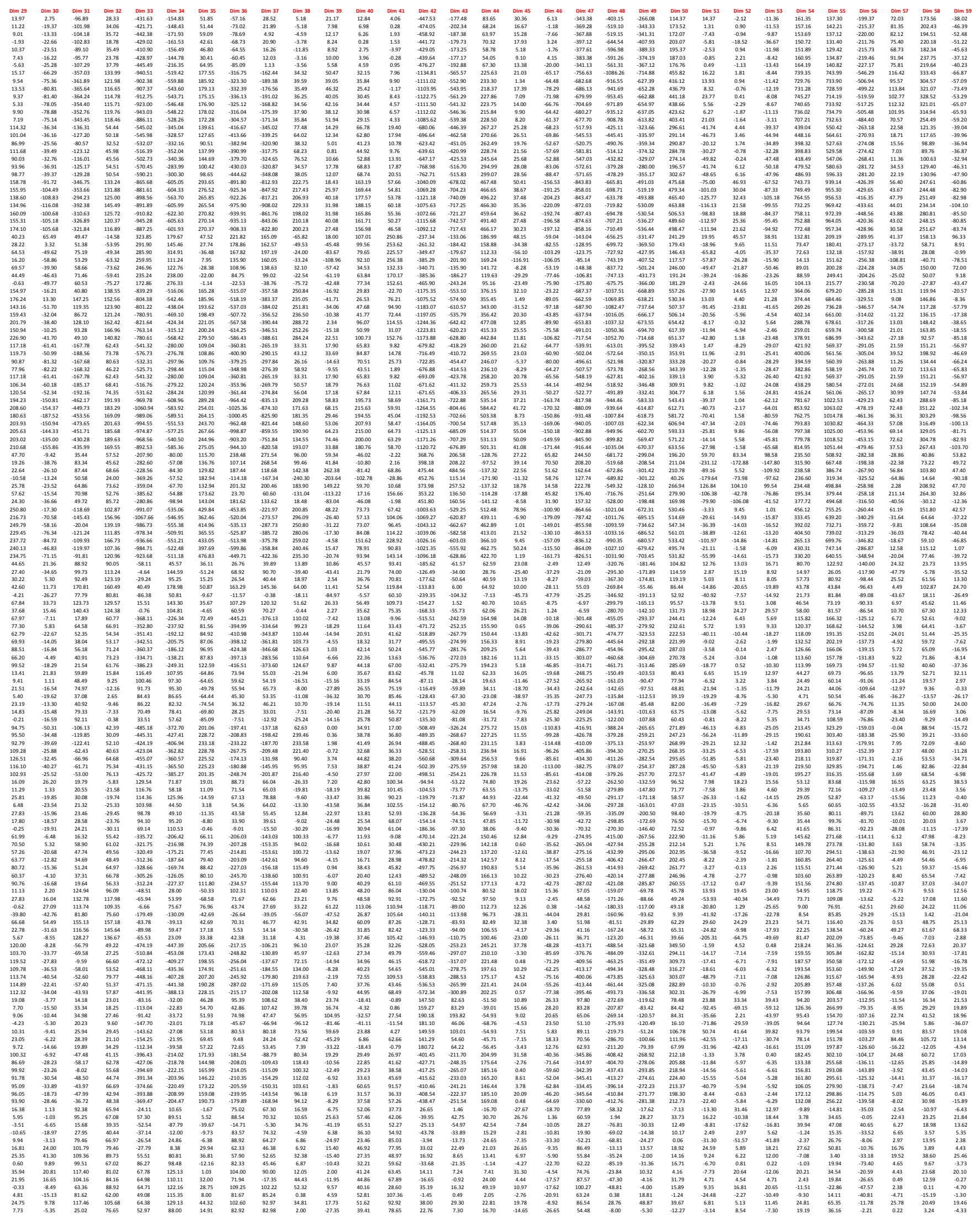


Table S5. Database for two min exposure to mouth and skin odor using handheld device.

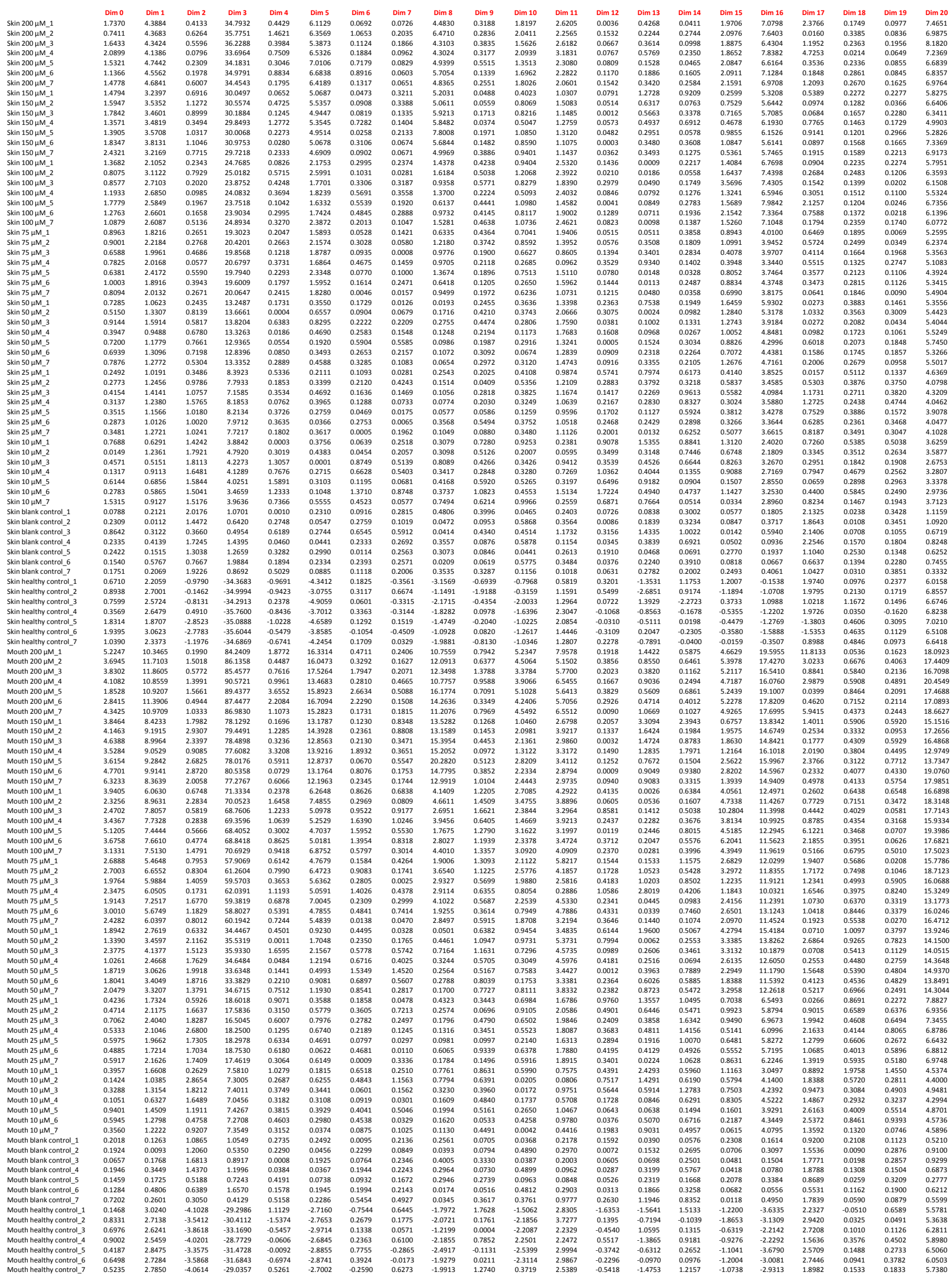




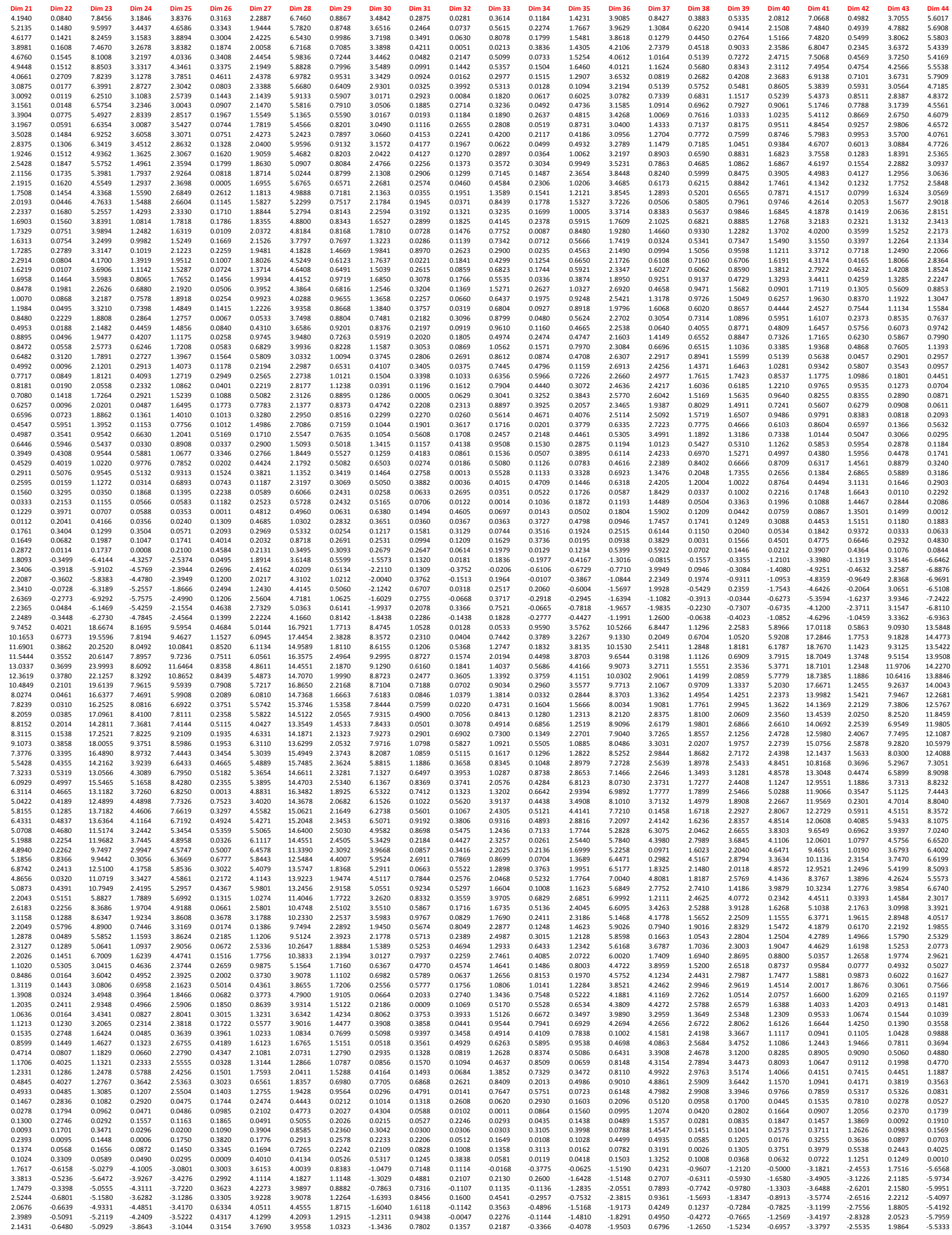




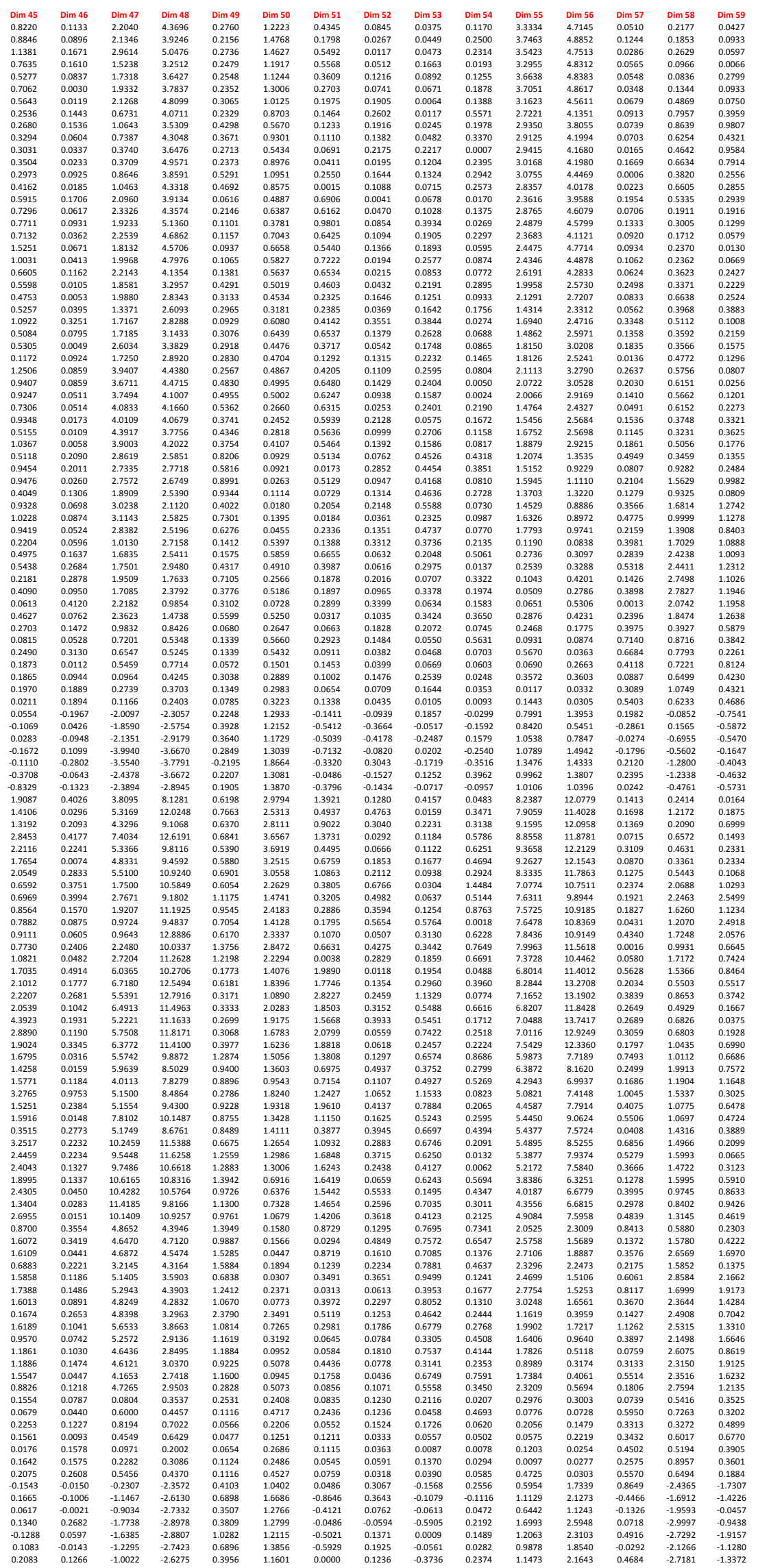

